\title{
Undesirable Flow Behavior in a Proposed Validation Data Set
}

\author{
ICONE 18
}

Richard W. Johnson

Hugh M. Mcllroy

Ryan C. Johnson

Daniel P. Christensen

The INL is a

U.S. Department of Energy

National Laboratory

operated by

Battelle Energy Alliance

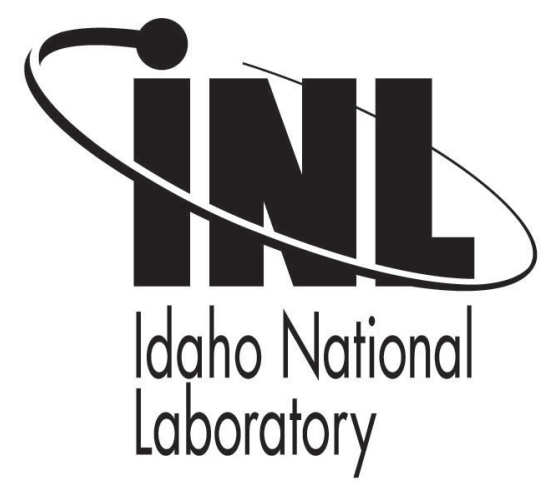

\section{May 2010}

This is a preprint of a paper intended for publication in a journal or proceedings. Since changes may be made before publication, this preprint should not be cited or reproduced without permission of the author. This document was prepared as an account of work sponsored by an agency of the United States Government. Neither the United States Government nor any agency thereof, or any of their employees, makes any warranty, expressed or implied, or assumes any legal liability or responsibility for any third party's use, or the results of such use, of any information, apparatus, product or process disclosed in this report, or represents that its use by such third party would not infringe privately owned rights. The views expressed in this paper are not necessarily those of the United States Government or the sponsoring agency. 


\section{ICONE18-29474}

\section{UNDESIRABLE FLOW BEHAVIOR IN A PROPOSED VALIDATION DATA SET}

\author{
Richard W. Johnson \\ Idaho National Laboratory \\ Idaho Falls, Idaho, USA \\ Rich.Johnson@inl.gov
}

\author{
Hugh M. Mcllroy \\ Idaho National Laboratory \\ Idaho Falls, Idaho, USA \\ Hugh.Mcllroy@inl.gov
}

\author{
Ryan C. Johnson \\ INL summer intern \\ Brigham Young University \\ Provo, Utah, USA
}

\author{
Daniel P. Christensen \\ INL summer intern \\ Utah State University \\ Logan, Utah, USA
}

\section{ABSTRACT}

The next generation nuclear plant (NGNP), whose development is supported by the U. S. Department of Energy, will be a very high temperature reactor (VHTR). The VHTR is a single-phase helium-cooled reactor that will provide helium at up to $800{ }^{\circ} \mathrm{C}$. The prospect of a coolant at these temperatures circulating in the reactor vessel demands that careful analysis be performed to ensure that excessively hot spots are not created and that sufficient mixing of the coolant is obtained. Computational fluid dynamics (CFD) coupled with heat transfer will be used to perform the desired analyses. However, primarily because of the imperfect nature of modeling turbulent flow, any CFD calculations used to perform nuclear reactor safety analysis must be validated against experimental data. Experimental data have been taken in a scaled section of the lower plenum of a prismatic VHTR at the matched index of refraction (MIR) facility at the Idaho National Laboratory. These data were taken with the intent that they be examined for use as validation data. A series of investigations have been conducted to assess the MIR data. Issues that have already been examined include the extent of the required computational domain, the outlet boundary condition, the inlet data and the effect of the turbulence model. One of the jets that flow into the model impacts on a wedge, which represents a portion of a hexagonal graphite block that is part of the inner wall of the lower plenum. The nature of the flow below this particular jet is such that a randomly varying recirculation zone is created. This recirculation zone is seen to change in size, causing a relatively long-time scale of motion or disturbance on the flow downstream. It is concluded that such a feature is undesirable in a validation data set, firstly because of its apparent random nature and, secondly, because to obtain an appropriate longtime average would be impractical because of the compute time required. It is found that by eliminating the first of the four inlet jets into the scaled model, the resulting recirculation zone is rendered stable.

\section{INTRODUCTION}

The core of the reference prismatic version of the VHTR consists of stacks of hexagonal blocks of graphite arranged in rings that will fill the reactor vessel. The core is held up by pillars that are located in the lower plenum. Helium flows through hundreds of coolant channels in the heated portion of the core and eventually into the lower plenum as jets. Figure 1 illustrates a cross-section of the lower plenum showing the support pillars (circles). The heated core is a ring of hexagonal blocks three blocks thick as seen in Fig. 1 (red circles). Layers of hexagonal graphite reflector blocks line the outer wall of the lower plenum as shown. A 1:6.55 scaled model of a narrow slice of the lower plenum was built to provide the geometry for a CFD validation data set. Figure 2 provides a plan view of the cross-section of the scaled model. The model is comprised of full and half cylindrical posts to represent the support pillars, four open jet inlets and a wedge. The wedge represents a hexagonal graphite block that lines the inner wall of the lower plenum. Figure 3 is a drawing of the scaled model showing the inflow jets ducts, the outflow plane and the coordinate system used.

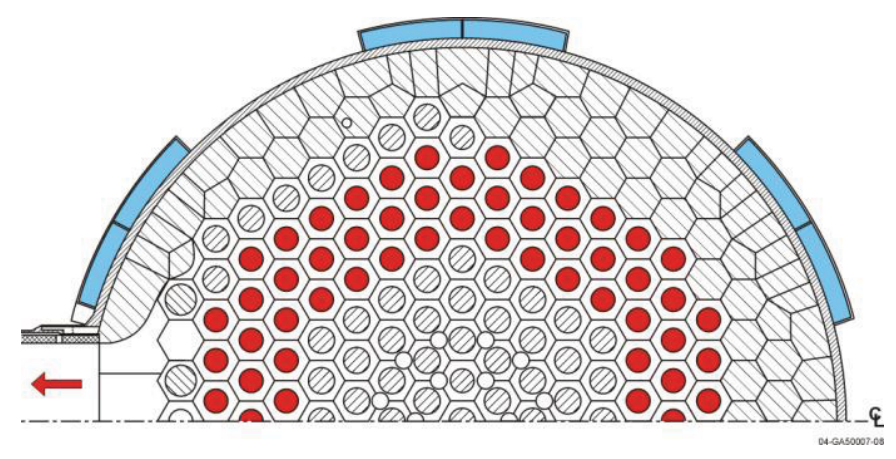

Figure 1. Plan view of the VHTR lower plenum. 
Inlet jets (open)

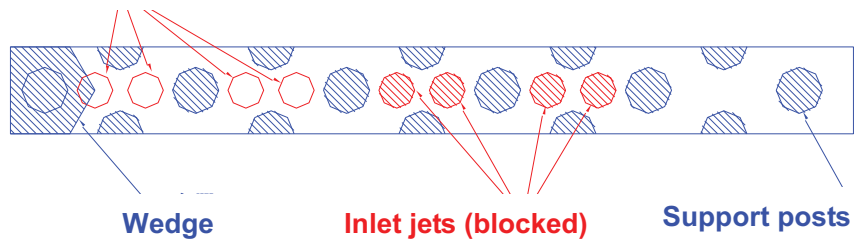

Figure 2. Plan view of the scaled model.

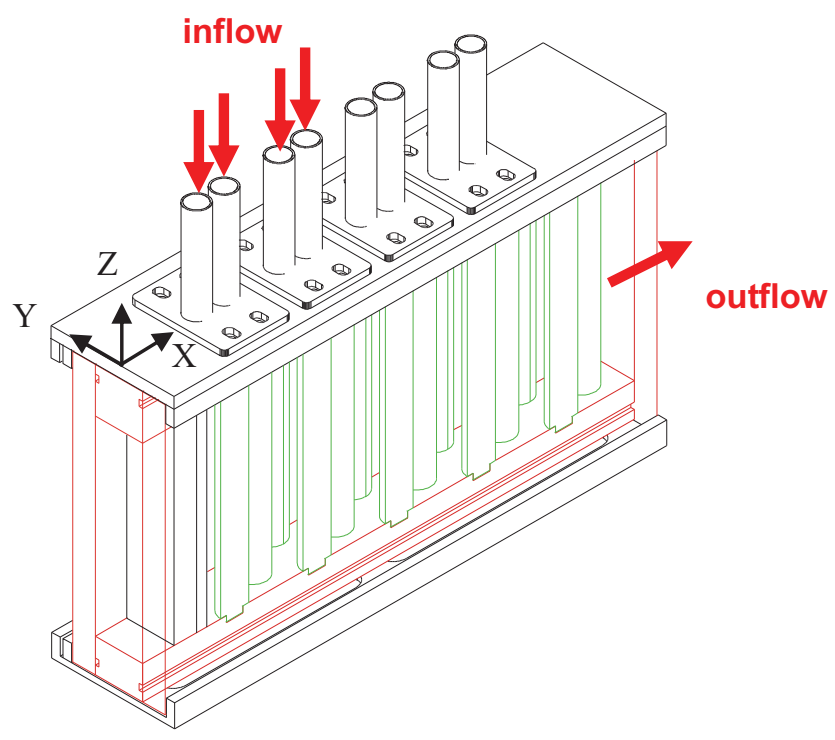

Figure 3. Isometric view of the scaled model.

Three-dimensional flow data were taken using stereo particle image velocimetry (PIV) [1] in the INL's matched index of refraction (MIR) facility. Data were taken for two flow regimes: inlet jet Reynolds numbers of 4300 and 12,400. The higher jet Reynolds number data are representative of the actual reactor at low $(10 \%)$ power and are the subject of the present investigations.

A series of studies have been made to examine the flow in the scaled model and similar geometry. Vortex-shedding flow in a staggered tube bank, Ref. [2], was a first step to being able to model turbulent flow in a similar nonstationary environment where the Reynolds-averaged Navier Stokes (RANS) equations are unsteady because of the vortex shedding and are actually ensemble-averaged. Hence, the approach is called a URANS approach. The Reynolds stress model (RSM) of turbulence, which solves a separate transport equation for each Reynolds stress, was used and compared favorably to the long-time averaged streamwise velocity of the existing experimental data from the ERCOFTAC database [3]. Similar calculations that assumed that the flow was steady were clearly in poorer agreement. The commercial code FLUENT [4] was used in the study.
A following study, Ref. [5], examined the domain that should be included in the CFD model. The scaled model was installed in the test section of the MIR test facility at the INL which has a $2 \mathrm{ft}$. by $2 \mathrm{ft}$. cross section. The working fluid, mineral oil, not only flowed through the jets and then through the scaled model, it also flowed around the outside of the model inside the test section of the MIR facility, albeit at a much lower velocity. Hence, there was the possibility to have vortexshedding at the ends of the $1 \mathrm{in}$. thick walls of the scaled model that might interfere with the flow exiting the scaled model. A two-dimensional study was made to compare results for computations that included the outer flow to those that only included the flow in the scaled model. It was found that the flow just upstream of the last pair of half cylinders was almost the same for the case of including the outer flow as for the case of computing only the inner flow if a pressure-outlet boundary condition was used. This led to confidence in computing just the inner flow for the 3-D case.

The next study, Ref. [6], involved 3-D computations in the geometry of the scaled model itself using commercial CFD code STARCCM+ [7]. Because it was found earlier [2] that the RSM turbulence model produced very good results for a similar nonstationary flow, it was used for this study also. The inlet conditions were applied at about $9.7 \mathrm{~mm}$ above the inlet plane to match PIV data that were provided at that level. Detailed inlet conditions for the three velocity components and the turbulent kinetic energy based on the PIV data as well as uniform inlet conditions based on data from the mass flow rotameters, which were used to measure and set the mass flow for each jet inlet, were applied. A pressure outlet condition was used. Calculations for the PIV-based inlet data eventually became steady, while those for the uniform conditions became nearly steady. Asymmetries were observed in both the calculations and in the MIR data, though not necessarily at the same locations. Numerical results for the region near the jets, especially jet 1 , which is located just above the point of the wedge, were in poor agreement with the data; results for the downstream regions showed much better agreement and similar trends for the long-time averaged velocities.

A subsequent study, Ref. [8], see also Ref. [9], examined the effects of using two-equation eddy viscosity turbulence models, starting from a constant versus a semi-converged steady computation and the effect of the inlet conditions. It was then realized that mass flow from the inlet conditions based on the detailed PIV data, when integrated over the inlet grid, were between 4 and $25 \%$ lower than the mass flow rotameter data; the latter data are considered to be the most accurate. The results indicated that the initial conditions did not make much difference in the results. However, the choice of turbulence model had a significant effect on the results. Results for the standard $k \sim \varepsilon$ two layer all $y^{+}$model were asymmetric in the long-time average and yielded unsteady results that were very regular with low amplitude oscillations. Results for the AbeKondoh-Nagano (AKN) $k \sim \varepsilon$ low $\operatorname{Re}$ all $y^{+}$model and the Menter shear-stress transport (SST) $k \sim \omega$ all $y^{+}$model showed 
symmetric long-time results but with unsteady oscillations with large variations in amplitude and with some interesting behavior wherein the ensemble mean (unsteady) velocities seemed to shift from one apparently semi-stable state to another. That is, velocities at specific locations would oscillate about a particular mean for a relatively long time, then shift to oscillate about a different mean with the frequency of shifting being apparently random. Again, long-time averaged velocities were seen to have poor agreement with the MIR data near the jet inlets, but much better agreement downstream.

It has been concluded that the best approach for the inlet conditions is to use the full height of the inlet ducts (about 88.5 $\mathrm{mm}$ ) with uniform inlet velocity. Just upstream of the inlet duct is a $90^{\circ}$ bend followed by flow straighteners and a turbulence inducing screen. The inlet flow is based on the mass flow rotameter data and a uniform turbulent kinetic energy that matches the MIR data at about $\mathrm{Z}=9.7 \mathrm{~mm}$ in the interior of the inlet ducts. The objective of the present investigation is to apply these "best inlet" conditions and then investigate the apparent presence of more than one semi-stable state in the calculations using the Menter shear-stress transport (SST) $k \sim \omega$ turbulence model that exhibits these features. Three dimensional CFD calculations have been carried out to perform these investigations.

\section{CFD MODEL}

The geometry of the scaled model has a width of 53.98 $\mathrm{mm}$, an overall length of $485.42 \mathrm{~mm}$, and a height of 217.50 $\mathrm{mm}$. The height of the inlet ports beyond a turbulencegenerating screen is $88.5 \mathrm{~mm}$. Figure 4 illustrates the CFD model/mesh that is based on the scaled model with green surfaces representing inlets, gray surfaces representing walls and the outlet plane red. The origin of the coordinate system is shown in Fig. 3.

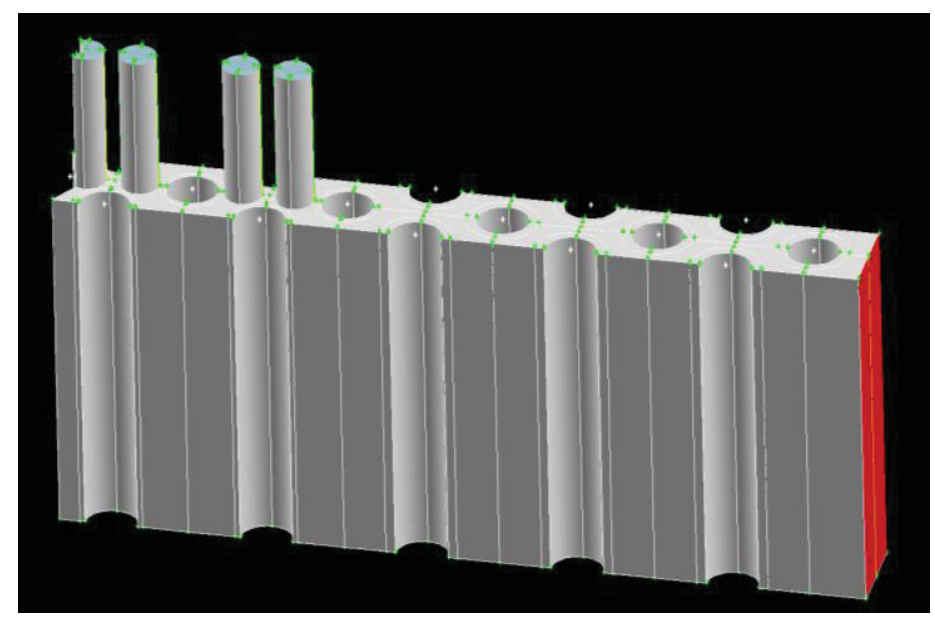

Figure 4. Geometry of the extruded 3-D mesh.

The mesh (grid a4) is constructed with 5.78 million hexahedral cells. The fineness of the cross-sectional mesh is based on the coarse mesh used in the two-dimensional study of
Ref. [5]. While this mesh is not necessarily fine-enough for validation purposes, it seems to be fine enough to investigate the main features of the flow, which is sufficient for present purposes. Figure 5 provides a closeup of the mesh showing two of the inlet ports and some of the half and full support posts. The 2-D mesh was extruded in the vertical $(Z)$ direction to create the model and the inlet ducts. The inlet ducts and model have 100 and 110 divisions in the vertical direction, respectively. Commercial mesh software GAMBIT 2.4.6, bundled with FLUENT [4], was used to create the mesh.

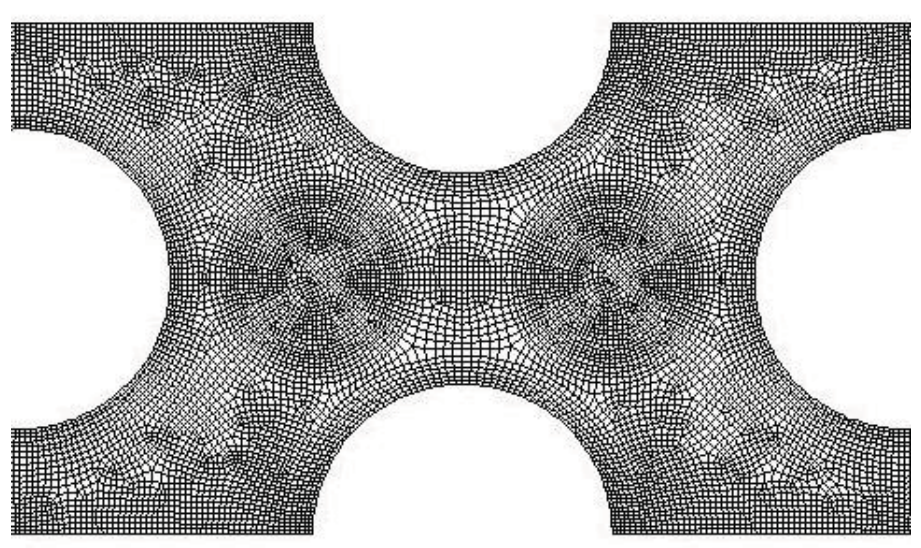

Figure 5. Closeup of fine 3-D mesh showing support posts and inlet jets $3 \& 4$.

Commercial CFD code STAR-CCM+ [7] is used for the 3$\mathrm{D}$ computations. Fluid properties are those of mineral oil at $23.3^{\circ} \mathrm{C}$. Second-order differencing is used for both spatial and implicit temporal discretization; the segregated solver is used. The pressure-outlet boundary condition is used at the outlet. The inlet vertical velocity is set to the bulk mean velocity consistent with measurements made with the mass flow rotameters at the MIR facility; the other components are set to zero. The inlet turbulent kinetic energy is set to $0.1 \mathrm{~J} / \mathrm{kg}$, which matches the measured turbulent kinetic energy in the interior of the inlet ducts at $Z=9.7 \mathrm{~mm}$ as measured by the PIV system. The inlet specific dissipation rate is set to $1.0 \mathrm{sec}^{-1}$. Initial conditions are set to uniform values throughout the domain. The time step is set to $2.0 \times 10^{-4} \mathrm{sec}$., with 20 iterations at each time step, which is sufficient to converge the residuals to well below $1 \times 10^{-4}$, which was found to be adequate [8]. The time step is adequate to fully resolve vortex-shedding time scales which are of the order of 0.03 seconds.

The primary calculations are based on the Menter shearstress transport (SST) $k \sim \omega$ all $y^{+}$turbulence model. The nearwall $\mathrm{y}^{+}$values range from about 1 to 20 , which include the viscous sublayer. This range is handled by the "all $\mathrm{y}^{+}$" wall treatment.

\section{RESULTS AND DISCUSSION}

Figure 6 illustrates locations of points and profiles for comparison purposes. A suffix will appear with the points/lines 
shown to indicate vertical location. Suffix ' $u$ ' indicates that the point is 'upward' or at $\mathrm{Z}=-0.07 \mathrm{~m}$, while ' $\mathrm{d}$ ' indicates that the line/point is 'downward' or at $Z=-0.15 \mathrm{~m}$. Because the scaled model is $0.217 \mathrm{~m}$ high, ' $\mathrm{u}$ ' and ' $\mathrm{d}$ ' are about one-third and twothirds the way down from the $\mathrm{Z}=0$ (inlet) plane.

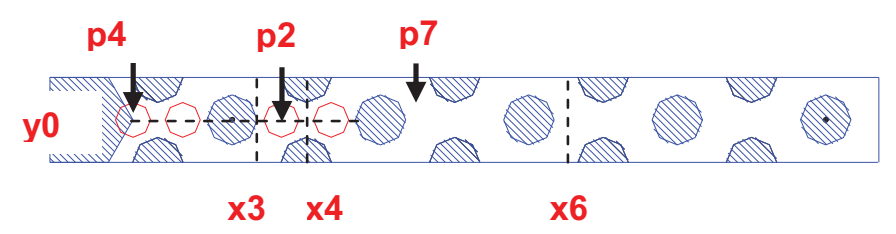

Figure 6. Locations of points and profiles.

The present study is a URANS study, discussed earlier, because the flow is unsteady not only because of the turbulence, but also because of vortex-shedding behind the full and half posts. To obtain the URANS equations, the dependent variables in the Navier-Stokes equations are first decomposed into an ensemble and a random component. (More details are given in Ref. [2]). For example, for the Z-component velocity:

$$
w=\langle w\rangle+w^{\prime}
$$

where $w$ is the instantaneous value, $\langle w\rangle$ is the ensemble average and $w^{\prime}$ is the random turbulent fluctuation. The velocities computed by the CFD code are the ensembleaveraged velocities. These velocities can be further decomposed into a long-time-averaged component and a coherent fluctuation. Here, the coherent fluctuation is assumed to be related to vortex-shedding. This decomposition is written as

$$
<w>=W+\widetilde{w}
$$

where $W$ is the long-time average or time mean of the ensemble average and $\widetilde{w}$ is the coherent fluctuation.

The velocity components for the present study are $u, v$ and $w$ in the $\mathrm{X}, \mathrm{Y}$ and $\mathrm{Z}$ directions, respectively. These variables are long-time-averaged by the CFD code by performing a running time-average during the course of the CFD run. The MIR data represent long-time-averaged quantities because they were averaged over a time period of 375 seconds [1]. However, the MIR data were taken at a very slow rate of only 2 to 3 frame pairs per second. Nevertheless, because of the long time period over which they were averaged, it is believed that sufficient information was used to provide representative long-time averages. Hence, comparisons between the MIR data and the CFD calculations are for long-time-averaged quantities.

Comparisons are made between earlier results, Ref. [8], where uniform inlet conditions, based on the same mass flow rates, were applied at inlets about $9.7 \mathrm{~mm}$ above the $\mathrm{Z}=0$ plane (grid a2) with turbulence intensity $=0.01$ and viscosity ratio $=$ $\mu_{t} / \mu=10$. Figure 7 compares results, both for the Menter shearstress transport (SST) $k \sim \omega$ all $y^{+}$model, for the time mean velocity $W$ at locations y0u and y0d. The signatures for the four jets are visible in the data for the upper location (Fig. 7a) and for the first two jets in the lower location (Fig. 7b); the second two jets have been swept away at the lower location.

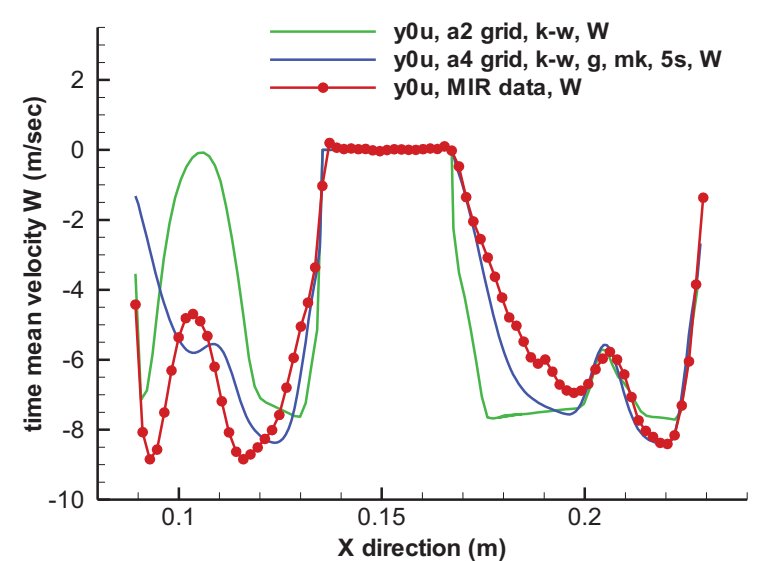

(a)

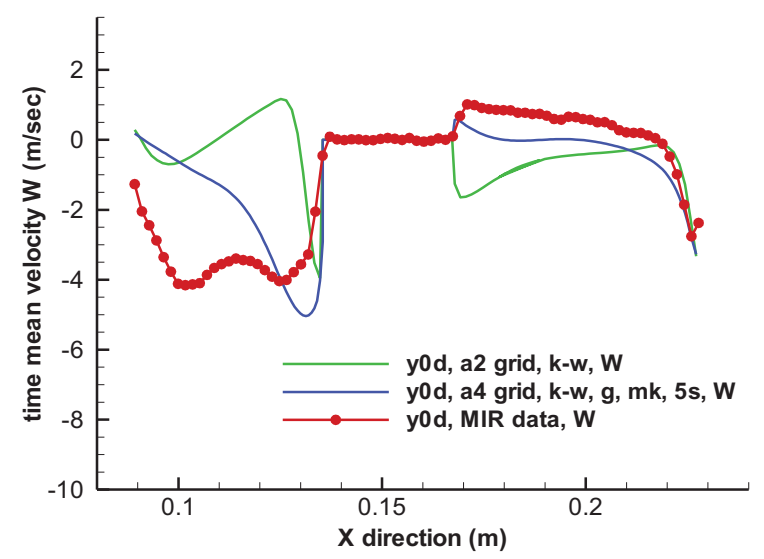

(b)

Figure 7. Time mean velocity $W$ at (a) y0u and (b) y0d.

Clearly, the results for the present case using the 'best inlet' conditions (with grid a4) are superior to the previous results using different turbulence inlet conditions at a different inlet height. These results show how predictions are poor below the first inlet jet but much better for the other three jet locations. In fact, results for $W$ below jet 4 (along y0u) coincide with the MIR data.

Figure 8 plots the time mean velocity $U$ at $x 3 \mathrm{u}$ and $\times 3 \mathrm{~d}$. At $\mathrm{x} 3 \mathrm{u}$, the flow is mostly vertical while at $\mathrm{x} 3 \mathrm{~d}$, the flow has turned toward the downstream outlet. The results for the 'best inlet' conditions are a definite improvement over the previous results. Also shown are the anomalous spikes in the MIR data, not seen in the calculations, and which may be artifacts of the unstable recirculation zone that is discussed below.

Figure 9 plots the time mean velocity $U$ at $x 4 \mathrm{u}$ and $\mathrm{x} 4 \mathrm{~d}$. At this location the flow accelerates as it passes between the two half cylinders. Again the 'best inlet' results are clearly better. The profile at $\mathrm{x} 4 \mathrm{~d}$ actually follows the MIR data quite closely.

Figure 10 illustrates results for time mean velocity $U$ for the two cases at $\mathrm{x} 6 \mathrm{u}$ and $\mathrm{x} 6 \mathrm{~d}$. At this location, the effects of the inlet jets have greatly diminished and the profiles are quite symmetric. While the $\mathrm{x} 6 \mathrm{u}$ profile for the 'best inlet' case is too high at the peaks relative to the MIR data, the profile for $x 6 \mathrm{~d}$ more closely follows the data. 


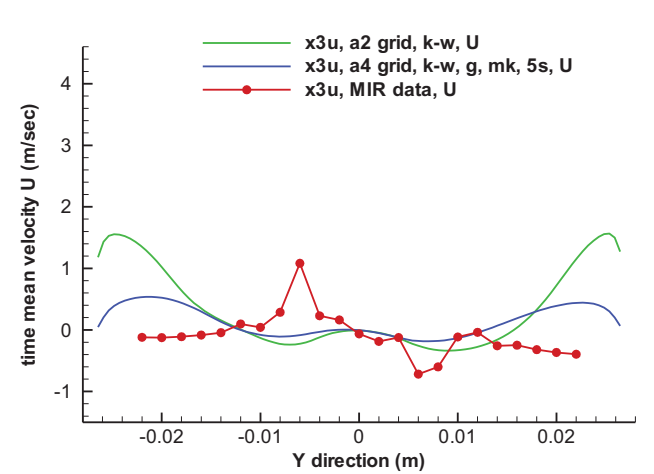

(a)

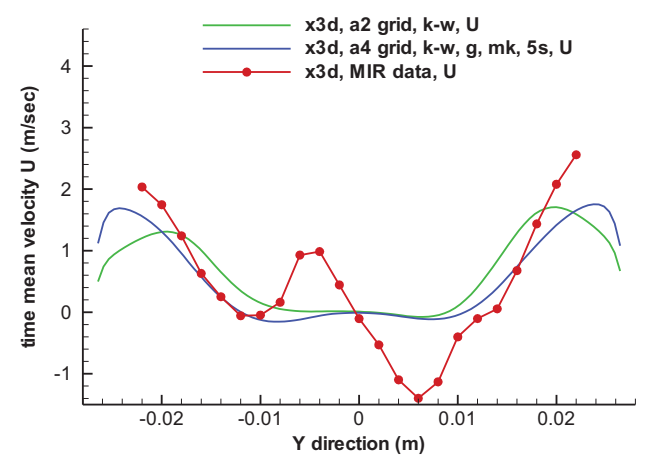

(b)

Figure 8. Time mean velocity $U$ at (a) $x 3 u$ and (b) $x 3 d$.

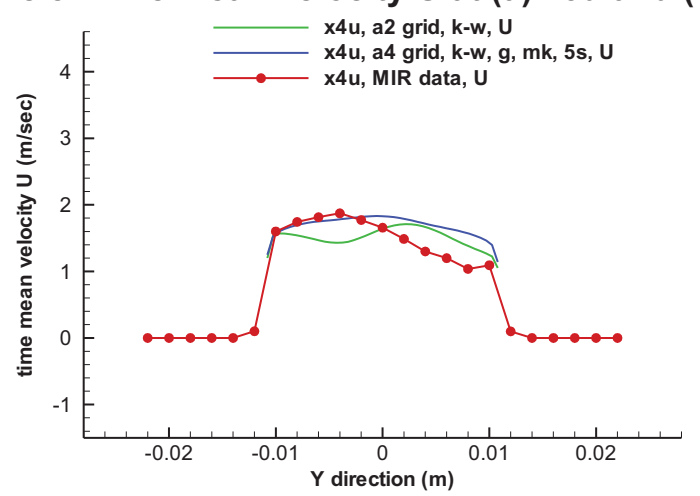

(a)

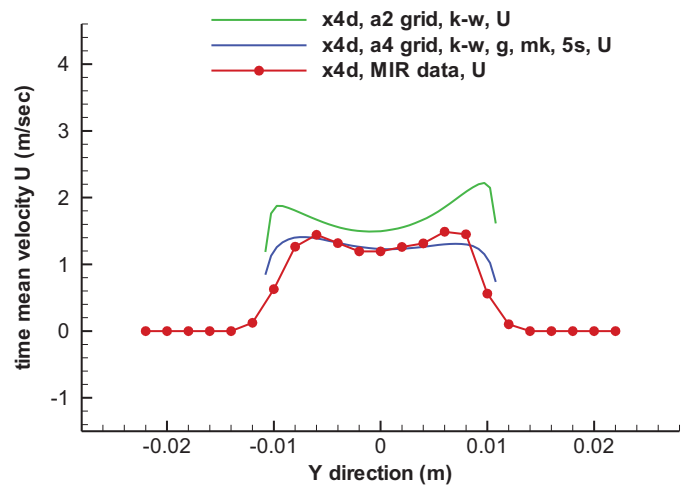

(b)

Figure 9. Time mean velocity $U$ at (a) $x 4 u$ and (b) $x 4 d$.

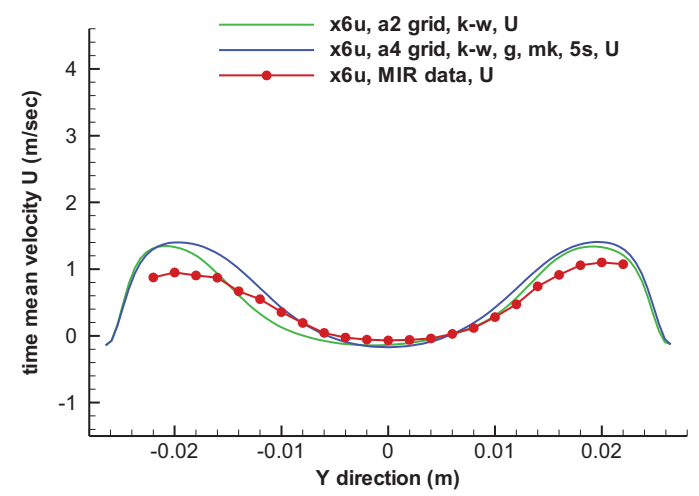

(a)

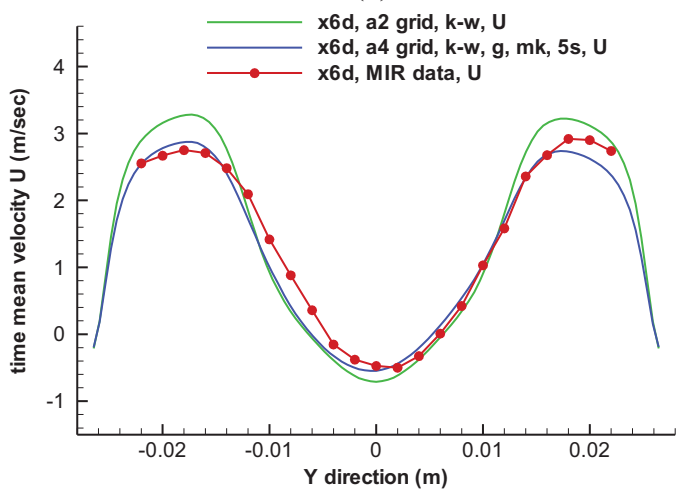

(b)

Figure 10. Time mean velocity $U$ at (a) $x 6 u$ and (b) x6d.

As has been seen in earlier studies, Refs. $[6,8,9]$, the agreement with the MIR data improves the further the profiles are from the inlet jets. Also, differences between the two sets of calculations are less for locations farther from the inlet jets, as expected.

Earlier experience using the RSM turbulence model [6], where the transients of the flow died out and the flow became steady for uniform inlet conditions and weakly unsteady for inlet conditions based on detailed PIV data, prompted that time traces of the unsteady ensemble-averaged velocity components be kept to keep track of the state of the unsteadiness, in particular, if there was unsteadiness related to vortex-shedding. Time traces were provided in Ref. [8] to examine the unsteadiness for particular turbulence models; the traces were dramitically different for the different models.

Figure 11 plots time traces at point $\mathrm{p} 7 \mathrm{~d}$ for the ensembleaverage velocity, $\langle u\rangle$, for calculations based on grid a2 (inlets at $\mathrm{Z}=9.7 \mathrm{~mm}$ ) and uniform inlet velocities for three twoequation turbulence models from Ref. [8]. These are the standard $k \sim \varepsilon$ two layer all $y^{+}$model, the Abe-Kondoh-Nagano (AKN) $k \sim \varepsilon$ low Re all $y^{+}$model and the Menter shear-stress transport (SST) $k \sim \omega$ all $y^{+}$model. Turbulence inlet conditions were set to the default values of turbulence intensity $=0.01$ and turbulent viscosity ratio $=\mu_{t} / \mu=10$ for these cases. 


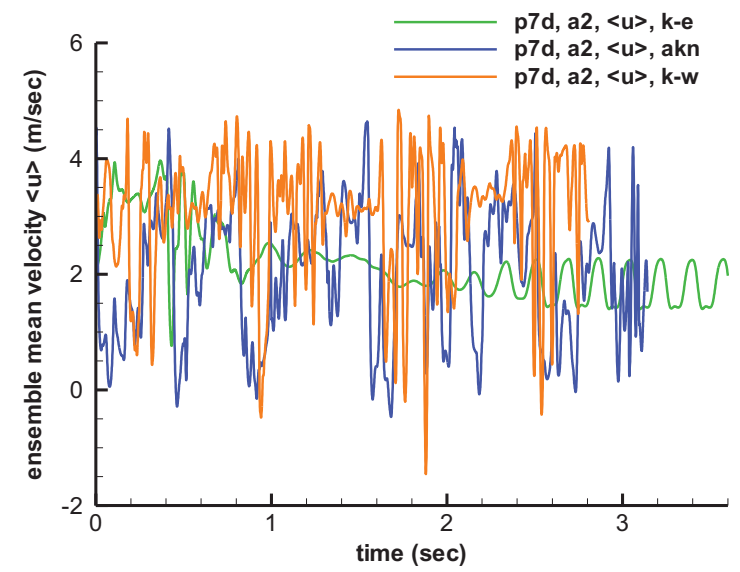

Figure 11. Time traces for 3 turbulence models.

From Fig. 11, it is seen that after about 2 seconds, the velocity $\langle u\rangle$ at point $\mathrm{p} 7 \mathrm{~d}$ is unsteady with a very regular oscillation for the standard $k \sim \varepsilon$ model. However, for the $k \sim \omega$ model, the time trace exhibits intervals of very high amplitude oscillations interspersed with intervals of very low amplitude oscillations. The time scale of the oscillations is of the order of vortex shedding as found in the earlier 2-D study [5], about $0.02 \mathrm{sec}$. The time scale of the intervals of high and low amplitude oscillations is on the order of $0.2 \mathrm{sec}$. The time trace for the AKN model exhibits large scale excursions with the small scale fluctuations superimposed onto the larger scale. In the previous study [8], it was surmised that there were some semi-stable states to which the numerical solution would gravitate based on these time traces.

Figure 12 illustrates time plots of the ensemble-mean velocity $\langle u\rangle$ at points $\mathrm{p} 7 \mathrm{u}$ and $\mathrm{p} 7 \mathrm{~d}$ for the present $k \sim \omega$ model using the best inlet conditions for the a4 grid. As can be seen, there are large excursions in the values at which the velocity oscillates for both traces. Also, the time scale of the excursion appears to be random. Again, this implies that there are relatively long-time-scale instabilities present in the flow.

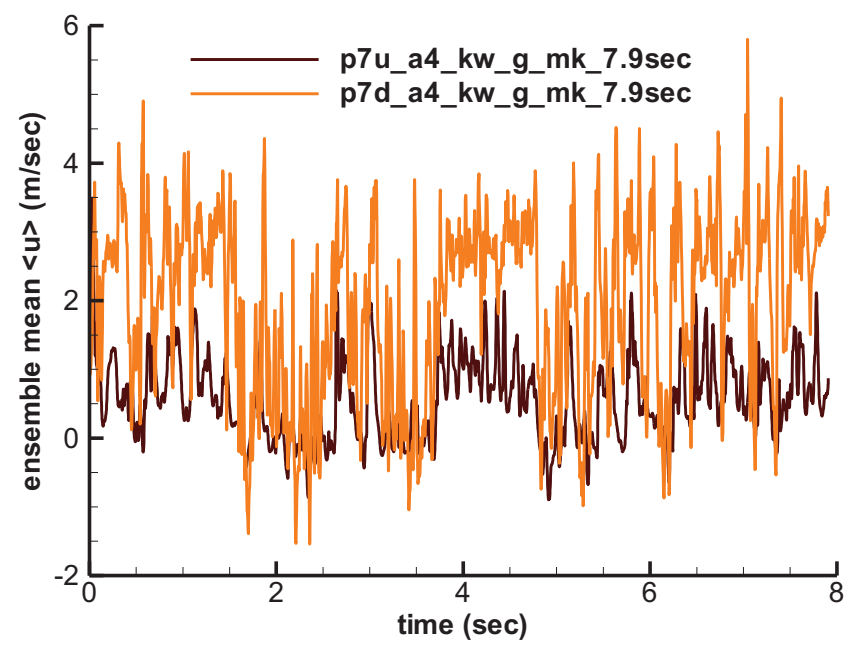

Figure 12. Time traces for $\langle u\rangle$ at $p 7 u$ and $p 7 d$.
Figure 13 illustrates time plots of the ensemble-mean velocity $\langle w\rangle$ at $\mathrm{p} 4 \mathrm{u}$ and $\mathrm{p} 2 \mathrm{u}$ for the same case as for Fig. 12 . Point $\mathrm{p} 4 \mathrm{u}$ is below jet 1 and $\mathrm{p} 2 \mathrm{u}$ is below jet 3. Again, large excursions are seen to occur in the time traces. In fact, the excursions are quite dramatic, swinging from about $-8 \mathrm{~m} / \mathrm{sec}$ to positive values of from $1(\mathrm{p} 2 \mathrm{u})$ to $4(\mathrm{p} 4 \mathrm{u}) \mathrm{m} / \mathrm{sec}$, that is, from a strong negative value to a positive value. Again, the excursion time scale is large and the length of the related time interval is apparently random. A long-time-scale instability is suggested by the flow dynamics.

A further examination is warranted to discover the nature of this long-time-scale instability. Figure 14 provides a streamline plot colored by velocity magnitude at $5 \mathrm{sec}$. compute time for the same case as for Figs. 12-13. A distinct recirculation zone is apparent in the lower left corner, just below the first jet. Figure 15 plots streamlines at 4.5 and 4.8 seconds for the same case. Clearly, the size of the recirculation zone is different for each of these times. This suggests that the instability is related to this recirculation zone.

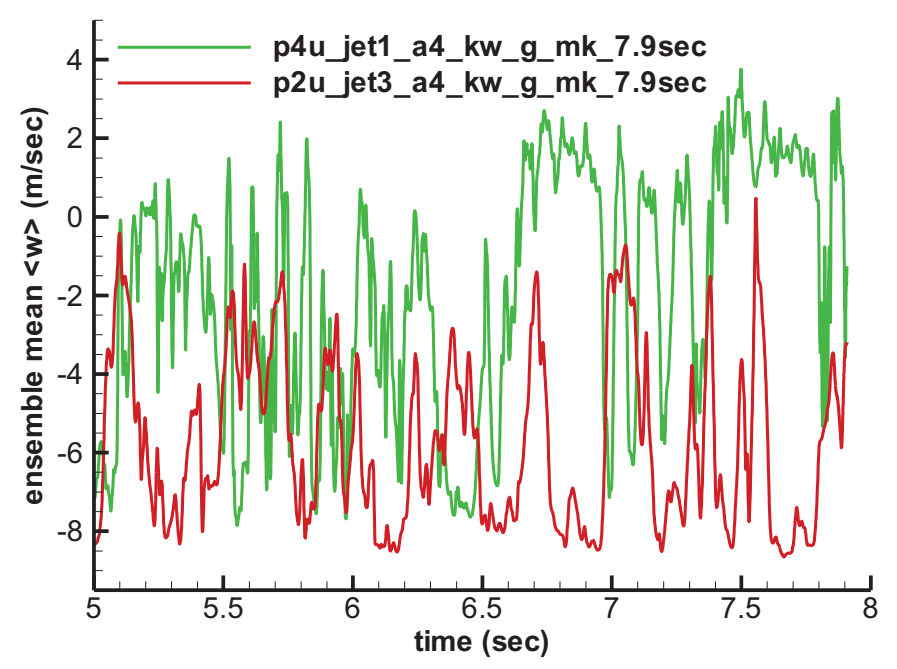

Figure 13. Time traces for $\langle w\rangle$ at $p 4 u$ and $p 2 u$.

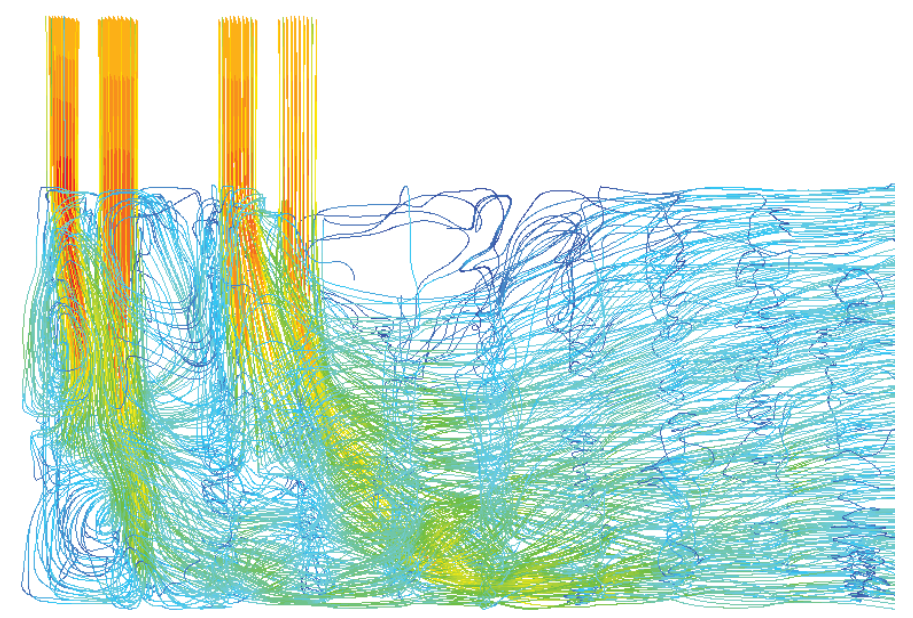

Figure 14. 3-D streamlines at $5 \mathrm{sec}$. 

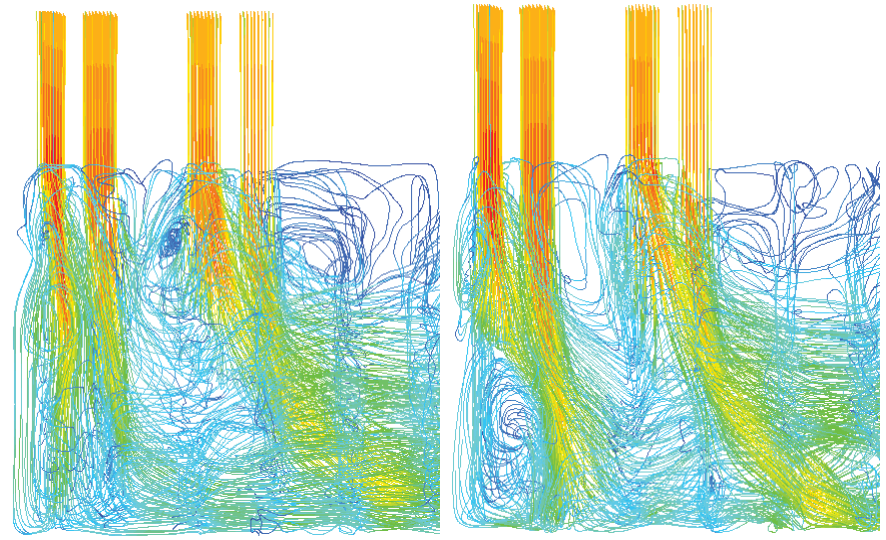

Figure 15. 3-D streamline plots at 4.5 and 4.8 seconds.

These results, of course, are from computations, and it has been shown that different turbulence models produce very different results. It is desirable to seek experimental confirmation that this lower recirculation zone is changing with time. A flow visualization study was performed where air was injected into jet 1 while mineral oil flowed into all four jets; a 15 second video was made. Figure 16 shows snapshots of the flow at four different times. It can be seen that the size of the lower left recirculation zone changes in size with time, that is, the detachment point at the top of the recirculation zone along the wall of the wedge is changing with time.
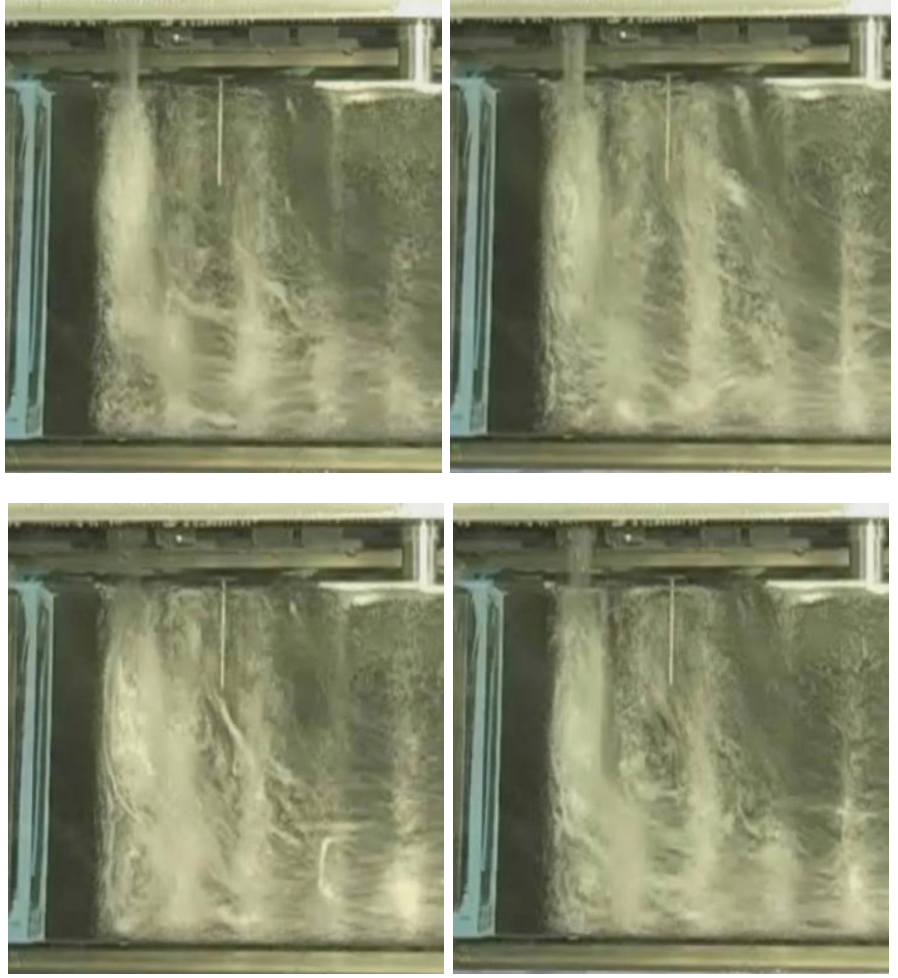

Figure 16. Flow visualization photos of the model at different times.
Figure 17 plots time traces of velocity component $u$ at points $\mathrm{p} 7 \mathrm{~d}$ and $\mathrm{p} 7 \mathrm{u}$ from the PIV data. These data were taken at a rate of only 2-3 times per second and hence do not represent time-accurate traces. Also, the turbulent fluctuation is still present, though of such a relatively small magnitude that it can be ignored for present purposes. Because the data are taken over such a long interval ( 375 seconds), they well capture the range of the data values of the velocity component. In fact, the ranges of the data captured for the two traces are very close to the ranges seen in the calculations of Fig. 12. The large fluctuation amplitudes are an indication of a possible instability.

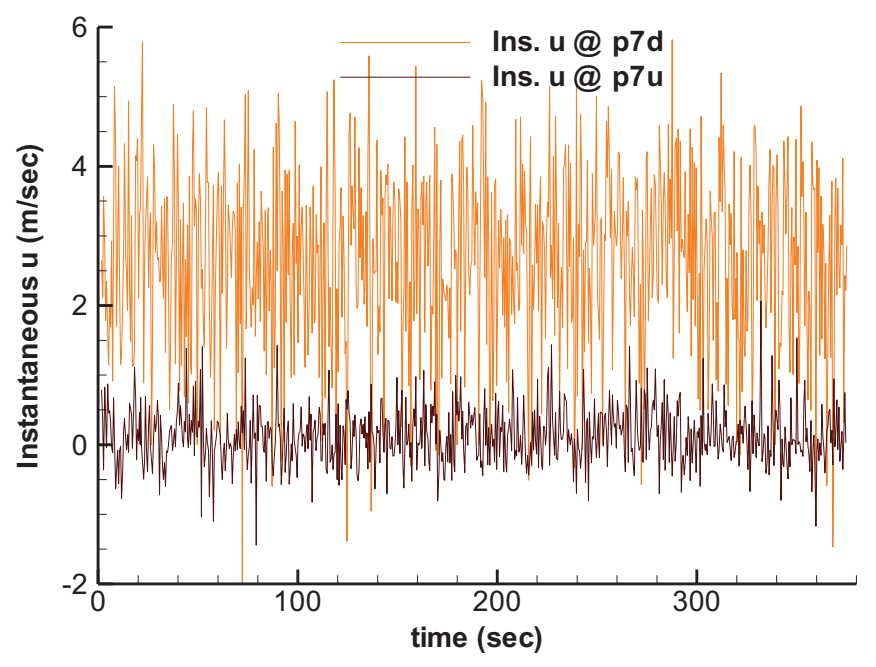

Figure 17. Traces of $u$ at $p 7 d$ and $p 7 u$ from MIR data.

Figure 18 presents a trace of instantaneous vertical velocity $w$ at point $\mathrm{p} 2 \mathrm{u}$, located within jet 3 from the MIR data. As shown, the range of the data varies dramatically from about -10 to $+2 \mathrm{~m} / \mathrm{sec}$, which range is only slightly larger than shown by the calculations in Fig. 13. Again, this is indicative of the presence of an instability.

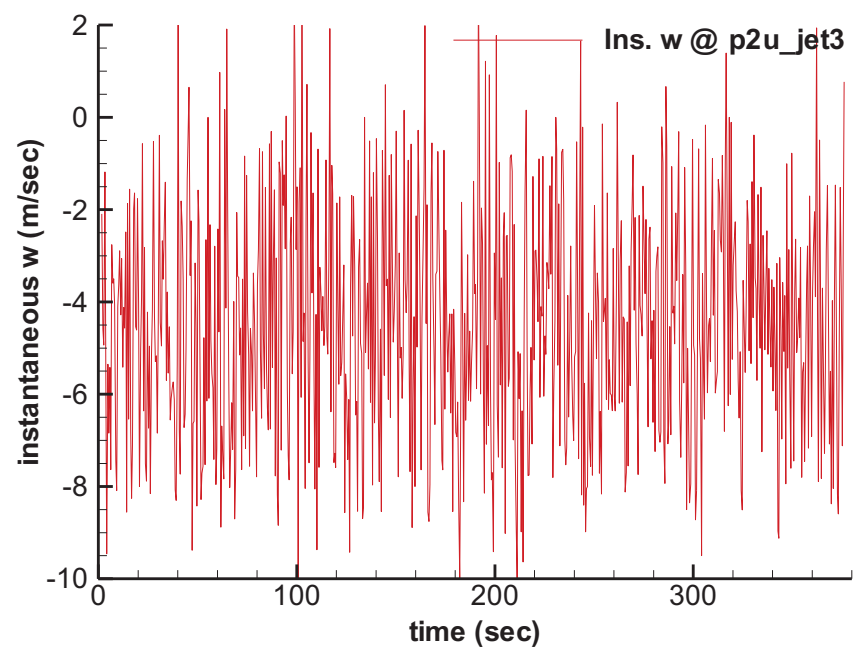

Figure 18. Trace of $w$ at $\mathrm{p} 2 \mathrm{u}$ from MIR data. 
Whether or not a jet inlet may actually be present above the corner of a hexagonal graphite block in the lower plenum of a gas-cooled reactor as envisioned in the design of the present scaled model, this configuration leads to an unstable flow situation wherein a recirculation zone changes size on an apparently random and relatively long time scale. Judging by the time traces plotted in Figs. 17 and 18, if there is a long-time average of the flow that is unchanging, it would be over such a long time, probably dozens of seconds, that it would be entirely impractical to perform a calculation for validation purposes. For the present, relatively coarse grid, the calculation of five seconds of flow time takes about seventeen days using over one hundred parallel cpus. Hence, the presence of the unstable recirculation zone makes the present geometry undesirable for a validation data set. That is, the randomly varying, long-timescale recirculation zone is an undesirable feature for a validation data set, not only because of the impracticability of computing it, but also because of its random nature. Rather, it seems much more appropriate to validate a CFD model with a problem close to that of the lower plenum flow, using a data set that does not include such a feature; one can then compute the flow with the undesirable feature with some confidence in the model because of the validation for the nearby problem.

It was thought that if the unstable recirculation zone below jet 1 is driven by the presence of jet 1 , perhaps it could be eliminated or rendered stable by eliminating jet 1 . Calculations have been made using the same grid (a4) and the same turbulence model as for the calculations reported in Figs. 12 15 , but with the first jet eliminated. The inlet is set to be a wall instead. The flow rates for the other jets are the same as before. Figure 19 plots time traces for the ensemble-average velocity $<w>$ at point $\mathrm{p} 2 \mathrm{u}$ (jet 3 ) for the same case as for Figs. $12-15$ and for the case of eliminating jet 1 . Instead of the dramatic excursions of velocity magnitude seen in the four-jet case, Fig. 18 , the no-jet-1 case shows no such swings.

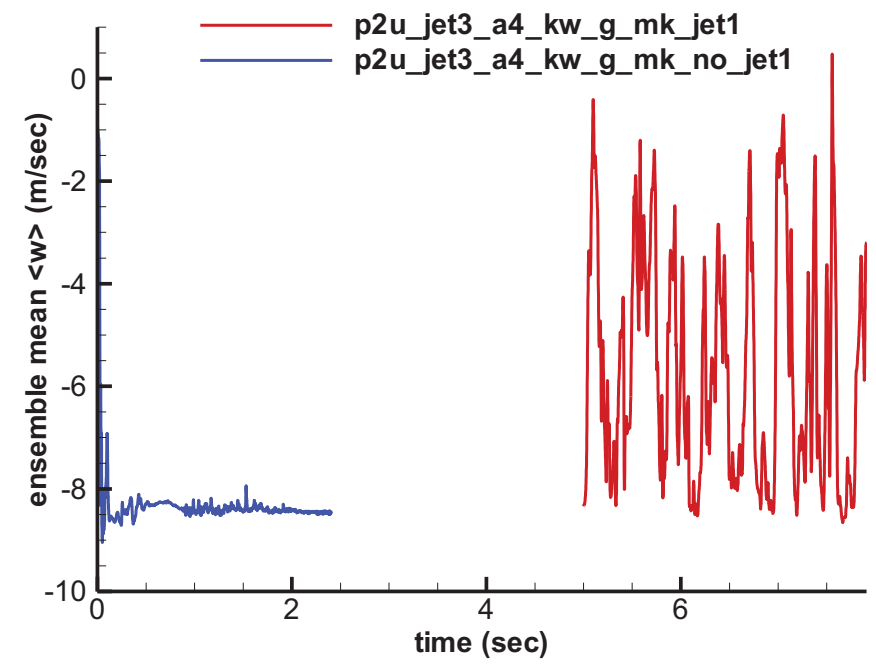

Figure 19. Time traces for $\langle w\rangle$ at $p 2 u$ (jet 3 ) for the case of all four jets and the case of jet 1 eliminated.
Figure 20 shows streamline plots for times 2.7, 3.0 and 3.28 seconds for the case of no-jet- 1 . As can be seen, there is a large recirculation zone below jet 2, but it apparently does not change in size as does the one in Figs. 14-15. The dramatic change in the range of fluctuating ensemble velocity as seen in Fig. 19, combined with the apparently unchanging recirculation zone as shown by Fig. 20 not only is a strong indication that the strong instability is not present for the no-jet-1 calculations, but that the instability is manifest by the dramatic excursions in velocity magnitude exhibited in the actual MIR data and the earlier calculations.
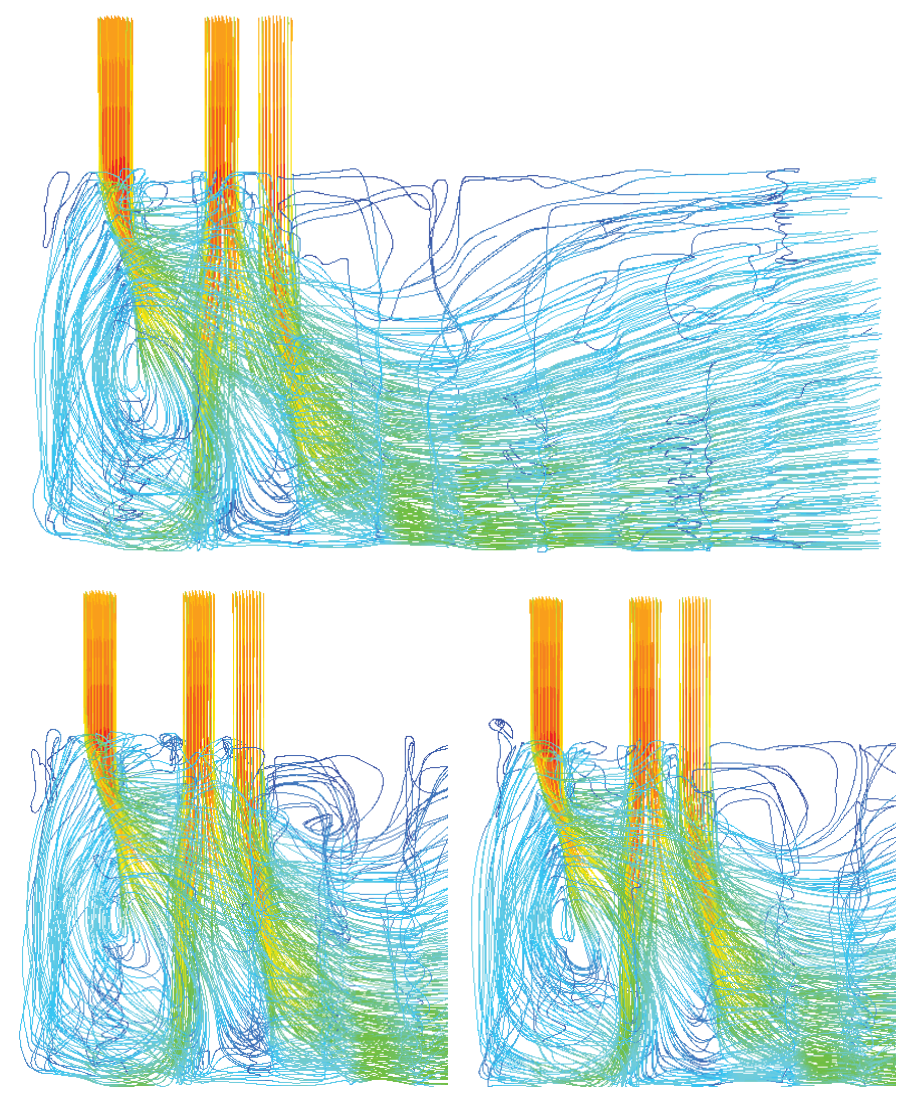

Figure 20. 3-D streamline plots at 2.7, 3.0 and 3.28 seconds for the case of no jet 1.

Comparing results for the no-jet-1 case compared to those including jet 1 strongly implies that the presence of jet 1 and its proximity to the upstream end of the model is the cause of the random long-time scale instability seen in the calculations and in the flow visualization study and in the MIR data. The simple solution to removing this undesirable flow feature is to plug jet 1 and only use jets 2-4. This will also allow a higher Reynolds number to be realized in the flow in the model; the current Reynolds number is significantly below that which is expected in the actual lower plenum flow (by about a factor of seven).

The previous study, Ref. 8, recommended that the inlet data for the flow be taken using PIV with the laser plane oriented perpendicular to the axes of the inlet ducts, rather than parallel as was done for the present MIR data. The present MIR data for 
the inlet conditions does not match inlet flow rotameter data for some of the jets. It is believed that the perpendicular orientation will provide much denser inlet data that should closely match the mass flow measured by the rotameters. With these two modifications, eliminating jet 1 and using a perpendicular laser plane, it is hoped that the two major problems with the present MIR data will be overcome, leading to the generation of excellent validation data.

\section{SUMMARY AND RECOMMENDATIONS}

Three-dimensional unsteady Reynolds-averaged NavierStokes (URANS) calculations have been made of a scaled model of a slice of the lower plenum of the prismatic next generation nuclear plant (NGNP). Earlier investigations showed some large discrepancies between inlet data measured by mass flow rotameter and detailed PIV instrumentation. Best inlet conditions were implemented in present calculations based on the more accurate rotameter data, and applied to the model just beyond where screens were installed in the inlet ducts to generate turbulence. The best inlet conditions are shown to produce better predictions than for other cases. However, time traces of the ensemble-averaged velocity components still showed strange behavior that would indicate some sort of instability in the flow field.

Further investigations were made of the experimental data and using calculations to understand the nature of the instability. It was found that a randomly varying recirculation zone occurs below the first inlet jet. The unstable recirculation zone is apparent from the presence of very large excursions of velocity at points as seen in both calculations and in the MIR data. Also, the size of the recirculation zone is seen to change based on streamline plots of the flow as well as flow visualization studies made of the apparatus. The recirculation zone is seen to be changing in size on a time scale of tenths of a second, far longer than the time scale of shedding vortices in the flow from the presence of cylindrical posts. Also, the length of the time interval appears to be random. Because of the relatively long time scale of the unstable recirculation zone, it would be impractical to extend computations long enough to attain acceptably long time averages. The random nature of the instability also combines to render it an undesirable feature to be present in a flow intended to be a validation data set. Calculations made for the case of eliminating the first inlet jet show a dramatic change in the nature of the flow field, with the recirculation zone becoming stable as indicated in time traces at particular points and in streamline flow plots.

It is recommended that new experimental data be taken for the same scaled model wherein the inlet data are taken using a laser plane for the PIV instrumentation that is perpendicular to the inlet duct axis to increase the density of the inlet data and make it more accurate. It is also recommended that the first inlet jet be plugged to render the recirculation zone stable. These modifications can be made without rebuilding the scaled model.

\section{NOMENCLATURE}

$\begin{array}{ll}\text { a2 } & 3.89 \mathrm{~m} \text {. cell grid with } 9.7 \mathrm{~mm} \text { inlets } \\ \text { a4 } & 5.78 \mathrm{~m} \text {. cell grid with } 88.5 \mathrm{~mm} \text { inlets } \\ \text { CFD } & \text { computational fluid dynamics } \\ \text { DOE } & \text { U. S. Department of Energy } \\ \text { INL } & \text { Idaho National Laboratory, Idaho Falls, ID } \\ k & \text { turbulent kinetic energy } \\ \text { MIR } & \text { matched index of refraction facility } \\ \text { NGNP } & \text { next generation nuclear plant } \\ \text { PIV } & \text { particle image velocimetry } \\ \text { RSM } & \text { Reynolds stress transport turbulence model } \\ \text { URANS } & \text { unsteady Reynolds-averaged Navier-Stokes } \\ \text { VHTR } & \text { very high temperature reactor } \\ y^{+} & \text {dimensionless wall distance } \\ \varepsilon & \text { turbulent energy dissipation rate } \\ \omega & \text { specific turbulent dissipation rate }\end{array}$

\section{ACKNOWLEDGMENTS}

This manuscript has been authored by Battelle Energy Alliance, LLC under Contract No. DE-AC07-05ID14517 with the U.S. Department of Energy.

\section{REFERENCES}

[1] McIlroy, H. M., D. M. McEligot and R. J. Pink, "Measurement of Flow Phenomena in a Lower Plenum Model of a Prismatic Gas-Cooled Reactor," Proceedings of the $16^{\text {th }}$ International Conference on Nuclear Engineering (ICONE-16), Orlando, May 11-15, 2008.

[2] Johnson, R. W., "Modeling Strategies for Unsteady Turbulent Flows in the Lower Plenum of the VHTR," Nuclear Engineering \& Design, 238, pp. 482-491, 2008.

[3] ERCOFTAC database. http://cfd.me.umist.ac.uk/erfcoftac/.

[4] FLUENT, version 6.3.26, 2008. FLUENT Inc., 10 Cavendish Court, Centerra Resource Park, Lebanon, NH, 03766.

[5] Johnson, R. W., "Development of a CFD Analysis Plan for the First VHTR Standard Problem," Proceedings of the $4^{\text {th }}$ International Topical Meeting on High Temperature Reactor Technology (HTR2008), Washington, D. C., Sept. 28 - Oct. 1, 2008.

[6] Johnson, R. W., "CFD Investigation of Experimental Data Proposed to be a Validation Data Set," Proceedings of the 17th International Conference on Nuclear Engineering (ICONE17), paper ICONE17-75604, Brussels, Belgium, July 12-16, 2009.

[7] STAR-CCM+, version 3.04, 2008, CD-adapco, 60 Broadhollow Road, Melville, NY 11747.

[8] Johnson, R. W., "Examination of a Proposed Validation Data Set Using CFD Calculations," Proceedings of ASME 2009 Fluids Engineering Division Summer Meeting, paper FEDSM2009-78317, Vail, Colorado, August 2-5, 2009.

[9] Johnson, R. W. and Schultz, R. R., Computational Fluid Dynamic Analysis of the VHTR Lower Plenum Standard Problem, Idaho National Laboratory external report INL/EXT-09-16325, July 2009. 